\title{
No KRAS mutations found in gastrointestinal stromal tumors (GISTs): molecular genetic study of 514 cases
}

\author{
Jerzy Lasota, Liqiang Xi, Tiffany Coates $^{1}$, RaShonda Dennis ${ }^{1}$, Moses O Evbuomwan ${ }^{2}$, \\ Zeng-Feng Wang, Mark Raffeld and Markku Miettinen
}

Laboratory of Pathology, National Cancer Institute (NCI), Bethesda, MD, USA

\begin{abstract}
Gastrointestinal stromal tumors (GISTs) are the most common mesenchymal tumors of the gastrointestinal tract. A great majority of GISTs is driven by pathological activation of KIT or platelet-derived growth factor receptor- $\alpha$ (PDGFRA), two closely related receptor tyrosine kinases. However, other genetic changes including gain-of-function BRAF mutations and loss of succinate dehydrogenase (SDH) complex activity have been identified in the subsets of KIT-, PDGFRA-wild type tumors. Genetic mutations affecting KIT, PDGFRA, BRAF and SDH complex functions are believed to be mutually exclusive events. Recently, KRAS codon 12 and 13 mutations were reported in a small subset of KIT or PDGFRA mutant GISTs. Moreover, in in vitro experiments, KIT mutants with concurrent KRAS mutation showed resistance to imatinib, a receptor tyrosine kinase inhibitor used in GIST treatment. The aim of this study was to evaluate a large cohort of GISTs to define frequency and clinical significance of KRAS mutations in this type of cancer. A well-characterized cohort of 514 GISTs was screened for KRAS mutations using Sanger sequencing $(n=450)$ and pyrosequencing $(n=64)$. In all, 350 gastric, 100 intestinal and 64 primary disseminated GISTs were analyzed. No KRAS mutations were found. In GIST, KRAS mutations are extremely rare if they exist $(<0.2 \%)$. Thus, mutational activation of KRAS does not seem to play any significant role in the development and progression of this type of cancer. Modern Pathology (2013) 26, 1488-1491; doi:10.1038/modpathol.2013.89; published online 24 May 2013
\end{abstract}

Keywords: GIST; KIT mutations; KRAS mutations; PDGFRA mutations; pyrosequencing; Sanger sequencing

Gastrointestinal stromal tumors (GISTs) are the most common mesenchymal tumors of the gastrointestinal (GI) tract. A great majority of GISTs is driven by pathological activation of KIT or platelet-derived growth factor receptor- $\alpha$ (PDGFRA), two closely related receptor tyrosine kinases. It has been shown that KIT and PDGFRA mutations are mutually exclusive genetic events. A small subset of GISTs lack KIT or PDGFRA mutations and are often referred to as KIT- and PDGFRA-wild-type (WT) GISTs. Recent studies revealed that WT GISTs represent a heterogeneous group of tumors, and classification into several subtypes including

\footnotetext{
Correspondence: Dr J Lasota, MD, Laboratory of Pathology, National Cancer Institute, 9000 Rockville Pike, Building 10, Room B1B47, Bethesda, MD 20892, USA.

E-mail: jerzy.lasota@nih.gov

${ }^{1}$ These authors were 2012 Summer NCI Interns and both contributed equally to this work.

${ }^{2} \mathrm{MOE}$ is NCI Post-Baccalaureate Fellow.

Received 7 January 2013; revised 4 April 2013; accepted 18 April 2013; published online 24 May 2013
}

succinate dehydrogenase (SDH)-deficient GISTs, neurofibromatosis 1 (NF1)-associated GISTs and BRAF-mutant GISTs. ${ }^{1}$

KIT and PDGFRA tyrosine kinase inhibitor, imatinib mesylate (STI571, commercially known as Gleevc/Glivec; http://www.novartis.com) has been successfully used to treat surgically inoperable primary and disseminated GISTs. Tumor sensitivity to imatinib differs among the patients and to some extent depends on GIST genotype. ${ }^{2}$

Recently, KIT and PDGFRA mutants with concurrent KRAS mutations were reported. Based on in vitro experiments, it was concluded that presence of KRAS mutation can predict tumor resistance to imatinib treatment. ${ }^{3}$ Thus, identification of such mutations might have clinical significance. A treatment employing other alternative tyrosine kinase inhibitors could be considered in such cases. ${ }^{4}$

The aim of this study was to evaluate a large cohort of well-characterized GISTs to define frequency and clinical significance of KRAS mutations in this type of cancer. 


\section{Materials and methods}

In all, 514 GISTs were selected for this study. All tumors were previously well characterized including immunohistochemical evaluation for DOG-1and KIT expression and mutation status of KIT and PDGFRA. ${ }^{1}$ Control cohort consisted of 10 randomly selected colon carcinomas, expected to contain a high percentage of KRAS mutations.

The formalin-fixed paraffin-embedded (FFPE) tumor samples were selected for DNA extraction. Five to ten 5- $\mu \mathrm{m}$ thick sections were deparaffinized with xylene, washed twice in ethanol, lyophilized and incubated with $10 \mu \mathrm{g} / \mu \mathrm{l}$ proteinase K (Roche Diagnostics, Indianapolis, IN) in Hirt-Buffer at $55^{\circ} \mathrm{C}$ for $24 \mathrm{~h}$. Subsequently, DNA was recovered using Maxwell16 robotic system and DNA IQ case work pro kit (Promega, Madison, WI).

\section{Sanger Sequencing}

KRAS codons 12 and 13, and flanking sequences were PCR amplified with forward: 5'-GTGTGACATG TTCTAATATAGTCA-3'and reverse: 5'-AGAATGGT CCTGCACCAGTAATAT- ${ }^{\prime}$ primers. PCR amplifications were performed with AmpliTaq Gold DNA polymerase (Applied Biosystems, Roche, Branchburg, NJ) following standard three-temperature PCR protocol with denaturing at $94{ }^{\circ} \mathrm{C}$, annealing at $45^{\circ} \mathrm{C}$ and extension at $72^{\circ} \mathrm{C}$. $50 \mu \mathrm{l}$ PCR reactions were evaluated on agarose gels. The 212-bp PCR products were extracted using QIAquick gel extraction kit (www.qiagen.com) and submitted for sequencing with above primers. Sanger direct sequencing was performed by MacrogenUSA (Rockville, MD). Obtained sequences were analyzed and aligned with KRAS reference sequence, NG_007524.1 (www.ncbi. nlm.nih.gov).

\section{Pyrosequencing}

KRAS codons 12, 13 and 61 were evaluated by pyrosequencing using a PyroMark Q24 instrument and the PyroMark Q24 KRAS v2.0 kit (Qiagen, Valencia, CA). PCR reactions were conducted in a total volume of $25 \mu \mathrm{l}$ containing genomic DNA template, $200 \mathrm{nM}$ of each forward and reverse primers, and $12.5 \mu \mathrm{l} 2 \mathrm{x}$ HotStarTaq Master Mix (Qiagen). COLD-PCR was used to enrich mutant alleles, which allows us to achieve a sensitivity to detect $2-3 \%$ mutant tumor cells. ${ }^{5}$ COLD-PCR conditions for KRAS were $95{ }^{\circ} \mathrm{C} 15 \mathrm{~min}, 10 \mathrm{x}\left(95^{\circ} \mathrm{C}\right.$ $\left.20 \mathrm{~s}, 53^{\circ} \mathrm{C} 30 \mathrm{~s}, 72^{\circ} \mathrm{C} 20 \mathrm{~s}\right), 72^{\circ} \mathrm{C} 5 \mathrm{~min}, 95^{\circ} \mathrm{C} 2 \mathrm{~min}$, $35 \mathrm{x}\left(95^{\circ} \mathrm{C} 20 \mathrm{~s}, 70^{\circ} \mathrm{C} 8 \mathrm{~min}, 80^{\circ} \mathrm{C} 3 \mathrm{~s}, 53^{\circ} \mathrm{C} 30 \mathrm{~s}\right.$, $\left.72{ }^{\circ} \mathrm{C} \quad 20 \mathrm{~s}\right), \quad 72{ }^{\circ} \mathrm{C} \quad 5 \mathrm{~min}, \quad 8^{\circ} \mathrm{C}$ hold. For the pyrosequencing reactions, $10 \mu \mathrm{l}$ of PCR product was immobilized on streptavidin-coated Sepharose beads (GE Healthcare) and processed according to the manufacturer's instructions.

\section{Results}

In all, 514 GISTs including 350 gastric, 100 intestinal and 64 primary disseminated tumors were analyzed in this study. In all the cases, the diagnosis was confirmed by distinctive histology, KIT and/or DOG-1 expression and typical genotype. ${ }^{1}$ There were 255 KIT mutants, 105 PDGFRA mutants and 117 KIT/ PDGFRA-WT GISTs. In 37 cases molecular genetic data were incomplete and 16 gastric KIT/PDGFRAWT GISTs were confirmed to represent SDH-deficient tumors. ${ }^{6}$ One small intestinal tumor was associated with NF1. Overall, 450 primary GISTs were screened for mutations using Sanger sequencing, the method used in the study, which reported presence of concurrent KRAS mutations in GISTs. ${ }^{3}$ In search for KRAS mutations acquired during tumor progression, 64 metastatic tumors were evaluated using highly sensitive pyrosequencing.

No mutations were found in KRAS codons 12 and 13 using standard PCR amplification and Sanger direct sequencing of PCR products. Also, pyrosequencing failed to detect KRAS mutations in codons 12, 13 or 61 in 64 highly malignant, primary disseminated GISTs. Based on these results, we have concluded that the frequency of KRAS mutants in GISTs is $<0.2 \%$. In contrast, KRAS mutations were found by both Sanger direct sequencing and pyrosequencing in 6 of 10 colon carcinomas independently evaluated by Sanger sequencing and pyrosequencing.

\section{Discussion}

KRAS, a cellular homolog of the Kirsten rat sarcoma virus oncogene, is a member of RAS family that includes HRAS, KRAS and NRAS. ${ }^{7,8}$ RAS genes encode closely related, 21-kDa membrane-bound, intracellular proteins. These proteins relay mitogenic growth signals into cytoplasm and nucleus, and possess intrinsic GTPase activity. Gainof-function mutations in RAS diminished normal GTPase function and caused alterations of RAS-RAF-MAPK signaling pathway leading to increased cell proliferation. ${ }^{9}$ In general, mutations affecting different components of this pathway were believed to represent mutually exclusive genetic events. ${ }^{10}$ However, a few exceptions have been reported in the literature recently. ${ }^{11}$

Gain-of-function KRAS mutations have been identified in different types of cancers, most commonly in colonic, pancreatic and pulmonary carcinomas and rarely in sarcomas. ${ }^{12} \mathrm{~A}$ few studies reported KRAS mutations in GI leiomyosarcomas. ${ }^{13,14}$ However, incomplete immunohistochemical and molecular genetic data of these tumors cast some doubt on these results. ${ }^{1}$ Also, previous studies have failed to detect KRAS mutations in GISTs, although they were based on relatively small number of cases. ${ }^{15-18}$ 
Table 1 Previously published studies that have failed to detect KRAS mutations in GISTs

\begin{tabular}{|c|c|c|c|}
\hline Study & Country & No. of tumors & Mutation detection method \\
\hline Agaimy et al ${ }^{15}$ & Germany, Switzerland & 69 (49 patients) & $\begin{array}{l}\text { Nested PCR } \\
\text { Sanger sequencing }\end{array}$ \\
\hline Martinho et al ${ }^{16}$ & Portugal & 26 & $\begin{array}{l}\text { Standard PCR } \\
\text { Single strand conformation polymorphism }\end{array}$ \\
\hline Daniels et $a l^{17}$ & Germany, The Netherlands & 78 & $\begin{array}{l}\text { Multiplex PCR } \\
\text { Single nucleotide probe extension assay }\end{array}$ \\
\hline Origone et $a 1^{18}$ & Italy & 81 & $\begin{array}{l}\text { Nested PCR } \\
\text { Sanger sequencing }\end{array}$ \\
\hline
\end{tabular}

More recently, concurrent KRAS mutations were reported in KIT- or PDGFRA-mutant GISTs. ${ }^{3}$ The study consisted of two GIST cohorts tested independently in Locarno, Switzerland and in Milan, Italy by standard PCR amplification and Sanger direct sequencing of PCR products. In the cohort from Locarno, 3 KRAS mutants (two gastric and one intestinal GIST) were identified among 60 analyzed tumors. Subsequent in vitro experiments showed that KRAS mutation can cause resistance of KIT-mutant GISTs to imatinib; ${ }^{3}$ however, the clinical significance of this observation hinges on a reasonable frequency of such mutations.

Thus, in this study, a large cohort of wellcharacterized primary GISTs was evaluated for KRAS mutations using similar mutation-screening strategy. Based on frequency of KRAS mutations reported in the GIST cohort from Locarno, we expected to identify at least 20 mutants among our cases; however, no KRAS mutations were found. In contrast, KRAS codon 12 and 13 mutants were identified in 6 of the 10 colon carcinomas used as a control cohort, in which about $50 \%$ cases were expected to have KRAS mutation.

Studies of colorectal metastatic carcinomas have shown that KRAS mutations could be acquired during tumor progression. ${ }^{19}$ No KRAS mutations have been yet reported in advanced or metastatic GISTs, although only a few such tumors were evaluated. Nevertheless mutational activation of PIK3CA, which is involved in KIT signaling pathway, was demonstrated in KIT/PDGFRA-WT, BRAF-mutant GIST at the time of progressive disease. ${ }^{20}$ Considering the latter observation, we have decided to evaluate the hypothesis that KRAS mutations could be seen in GIST during tumor progression. Sixty-four metastatic GISTs were screened for KRAS mutations using COLD-PCR and pyrosequencing, a methodology of higher analytical sensitivity than Sanger sequencing; ${ }^{21}$ however, no KRAS mutations were found.

A major difference in frequency of KRAS mutants between our and recently published study ${ }^{3}$ is difficult to explain. Further investigations are necessary to confirm that some GISTs might have unique KRAS genotype as reported by the group from Ticino, Switzerland, although based on current $(n=514)$ and previous studies $(n=254)$ it seems that such mutants are extremely rare (Table 1).
Proper characterization of genetic alteration in human cancers and reproducibility of research findings is essential for the design and improvement of preclinical and clinical cancer studies. ${ }^{22}$ In arriving era of next generation sequencing, mutational profiling of GIST will be based on simultaneous evaluation of many genes from various signaling pathways. This strategy might lead to significant accumulation of data on mutational activation of various genes involved in KIT-signaling pathway including KRAS and validate studies based on Sanger sequencing.

\section{Acknowledgements}

This work was supported in part by NCI's Intramural Research Program.

\section{Disclosure/conflict of interest}

The authors declare no conflict of interest.

\section{References}

1 Miettinen M, Lasota J. Histopathology of gastrointestinal stromal tumor. J Surg Oncol 2011;104: 865-873.

2 Heinrich MC, Owzar K, Corless CL, et al. Correlation of kinase genotype and clinical outcome in the North American Intergroup Phase III Trial of imatinib mesylate for treatment of advanced gastrointestinal stromal tumor: CALGB 150105 Study by Cancer and Leukemia Group B and Southwest Oncology Group. J Clin Oncol 2008;26:5360-5367.

3 Miranda C, Nucifora M, Molinari F, et al. KRAS and BRAF mutations predict primary resistance to imatinib in gastrointestinal stromal tumors. Clin Cancer Res 2012;18:1769-1776.

4 Demetri GD. Differential properties of current tyrosine kinase inhibitors in gastrointestinal stromal tumors. Semin Oncol 2011;38(Suppl 1):S10-S19.

5 Li J, Wang L, Mamon $\mathrm{H}$, et al. Replacing PCR with COLD-PCR enriches variant DNA sequences and redefines the sensitivity of genetic testing. Nat Med 2008;14:579-584.

6 Miettinen M, Wang ZF, Sarlomo-Rikala M, et al. Succinate dehydrogenase-deficient GISTs: a clinicopathologic, immunohistochemical, and molecular 
genetic study of 66 gastric GISTs with predilection to young age. Am J Surg Pathol 2011;35:1712-1721.

7 Parada LF, Tabin CJ, Shih C, et al. Human EJ bladder carcinoma oncogene is homologue of Harvey sarcoma virus ras gene. Nature 1982;297:474-478.

8 Der CJ, Krontiris TG, Cooper GM. Transforming genes of human bladder and lung carcinoma cell lines are homologous to the ras genes of Harvey and Kirsten sarcoma viruses. Proc Natl Acad Sci USA 1982;79:3637-3640.

9 Rodriguez-Viciana P, Tetsu O, Oda K, et al. Cancer targets in the ras pathway. Cold Spring Harbor Symposia and Qantitative Biology Vol. LXX (Cold Sprin Harbor Laboratory Press 2005.

10 Jebar AH, Hurst CD, Tomlinson DC, et al. FGFR3 and Ras gene mutations are mutually exclusive genetic events in urothelial cell carcinoma. Oncogene 2005;24:5218-5225.

11 Benesova L, Minarik M, Jancarikova D, et al. Multiplicity of EGFR and KRAS mutations in non-small cell lung cancer (NSCLC) patients treated with tyrosine kinase inhibitors. Anticancer Res 2010;30:1667-1671.

12 Fernandez-Medarde A, Santos E. Ras in cancer and developmental diseases. Genes Cancer 2011;2:344-358.

13 Yoo J, Robinson RA, Lee JY. H-ras and K-ras gene mutations in primary human soft tissue sarcoma: concomitant mutations of the ras genes. Mod Pathol 1999;12:775-780.

14 Hill MA, Ch Gong, Casey TJ, et al. Detection of K-ras mutations in resected primary leiomyosarcoma. Cancer Epidemiol Biomarkers Prev 1997;6:1095-1100.

15 Agaimy A, Terracciano LM, Dirnhofer S, et al. V600E BRAF mutations are alternative early molecular events in a subset of KIT/PDGFRA wild-type gastrointestinal stromal tumours. J Clin Pathol 2009;62:613-616.

16 Martinho O, Gouveia A, Viana-Pereira $\mathrm{M}$, et al. Low frequency of MAP kinase pathway alterations in KIT and PDGFRA wild-type GISTs. Histopathology 2009;55:53-62.

17 Daniels M, Lurkin I, Pauli R, et al. Spectrum of KIT/PDGFRA/BRAF mutations and Phosphatidylinositol-3-Kinase pathway gene alterations in gastrointestinal stromal tumors (GIST). Cancer Lett 2011;312: 43-54.

18 Origone $\mathrm{P}$, Gargiulo S, Mastracci L, et al. Molecular characterization of an Italian series of sporadic GISTs. Gastric Cancer; advance online publication, 5 January 2013 [e-pub ahead of print].

19 Otsuka K, Satoyoshi R, Nanjo H, et al. Acquired/ intratumoral mutation of KRAS during metastatic progression of colorectal carcinogenesis. Oncology Letters 2012;3:649-653.

20 Falchook GS, Trent JC, Heinrich MC, et al. BRAF mutant gastrointestinal stromal tumor: first report of regression with BRAF inhibitor dabrafenib (GSK2118436) and whole exomic sequencing for analysis of acquired resistance. Oncotarget 2013; 4:310-315.

21 Tsiatis AC, Norris-Kirby A, Rich RG, et al. Comparison of Sanger sequencing, pyrosequencing, and melting curve analysis for the detection of KRAS mutations: diagnostic and clinical implications. J Mol Diagn 2010;12:425-432.

22 Begley CG, Ellis LM. Drug development: raise standards for preclinical cancer research. Nature 2012; 483:531-533. 